Jonathan A. Sherratt · Mark A.J. Chaplain

\title{
A new mathematical model for avascular tumour growth
}

Received: 28 January 2000 / Revised version: 7 November 2000 /

Published online: 21 August 2001 - (C) Springer-Verlag 2001

\begin{abstract}
The early development of solid tumours has been extensively studied, both experimentally via the multicellular spheroid assay, and theoretically using mathematical modelling. The vast majority of previous models apply specifically to multicell spheroids, which have a characteristic structure of a proliferating rim and a necrotic core, separated by a band of quiescent cells. Many previous models represent these as discrete layers, separated by moving boundaries. Here, the authors develop a new model, formulated in terms of continuum densities of proliferating, quiescent and necrotic cells, together with a generic nutrient/growth factor. The model is oriented towards an in vivo rather than in vitro setting, and crucially allows for nutrient supply from underlying tissue, which will arise in the twodimensional setting of a tumour growing within an epithelium. In addition, the model involves a new representation of cell movement, which reflects contact inhibition of migration. Model solutions are able to reproduce the classic three layer structure familiar from multicellular spheroids, but also show that new behaviour can occur as a result of the nutrient supply from underlying tissue. The authors analyse these different solution types by approximate solution of the travelling wave equations, enabling a detailed classification of wave front solutions.
\end{abstract}

\section{Introduction}

Solid tumours develop initially as a single mass of cells. These divide more rapidly than the cells around them because of a proliferative advantage caused by mutation, and a number of genetic pathways responsible for these mutations have been identified over the last decade (see Grander (1998) and Weinberg (1991) for reviews). This initial phase of avascular growth leads to a non-metastatic tumour, which may remain dormant for a long period, with subsequent tumour growth depending other mutations that enable the tumour to acquire its own blood supply (see Henry (1999) and Chaplain \& Anderson (1996) for reviews).

The very early stages of tumour growth are rarely seen clinically because of the small size of the cell masses. However, this early growth has been well studied in vitro, via the multicellular spheroid system. This involves a ball of cells being

J.A. Sherratt: Centre for Theoretical Modelling in Medicine, Department of Mathematics, Heriot-Watt University, Edinburgh EH14 4AS, UK. e-mail: jas @ma . hw . ac . uk

M.A.J. Chaplain: Centre for Nonlinear Systems in Biology, Department of Mathematics, University of Dundee, Dundee DD1 4HN, UK. e-mail: chapla in@mcs . dundee . ac . uk

Key words or phrases: Avascular tumour - Reaction-diffusion - Cancer - Travelling wave - Multicellular spheroid 
grown in a liquid medium containing appropriate nutrients, initiated by a seed cell taken from a tumour cell line; the cell mass can grow up to a diameter of several $\mathrm{mm}$. This assay was introduced by Sutherland and coworkers in the early 1970s (Sutherland et al., 1971; see also the review article by Sutherland, 1988), and has been used extensively to study internal properties of the spheroids, to investigate subsequent stages of tumour progression, and to test therapeutic strategies (see Kunz-Schughart et al. (1998) and Hamilton (1998) for recent reviews). Multicellular spheroids have a well-established characteristic structure (illustrated in Figure 1). There is an outer rim of proliferating cells (a few hundred $\mu \mathrm{m}$ thick) and an inner core of dead cells. Between these is a layer of quiescent cells, which are not dividing but are alive, and can begin dividing again if environmental conditions change. Such quiescent cells are very important, since many cancer therapies, including radiotherapy, specifically target dividing cells, so that a quiescent population is central to treatment failure (Schwachofer et al., 1991).

The reason for cells in a multicellular spheroid becoming quiescent and then dying has been studied extensively, but there is still no definitive answer. Levels

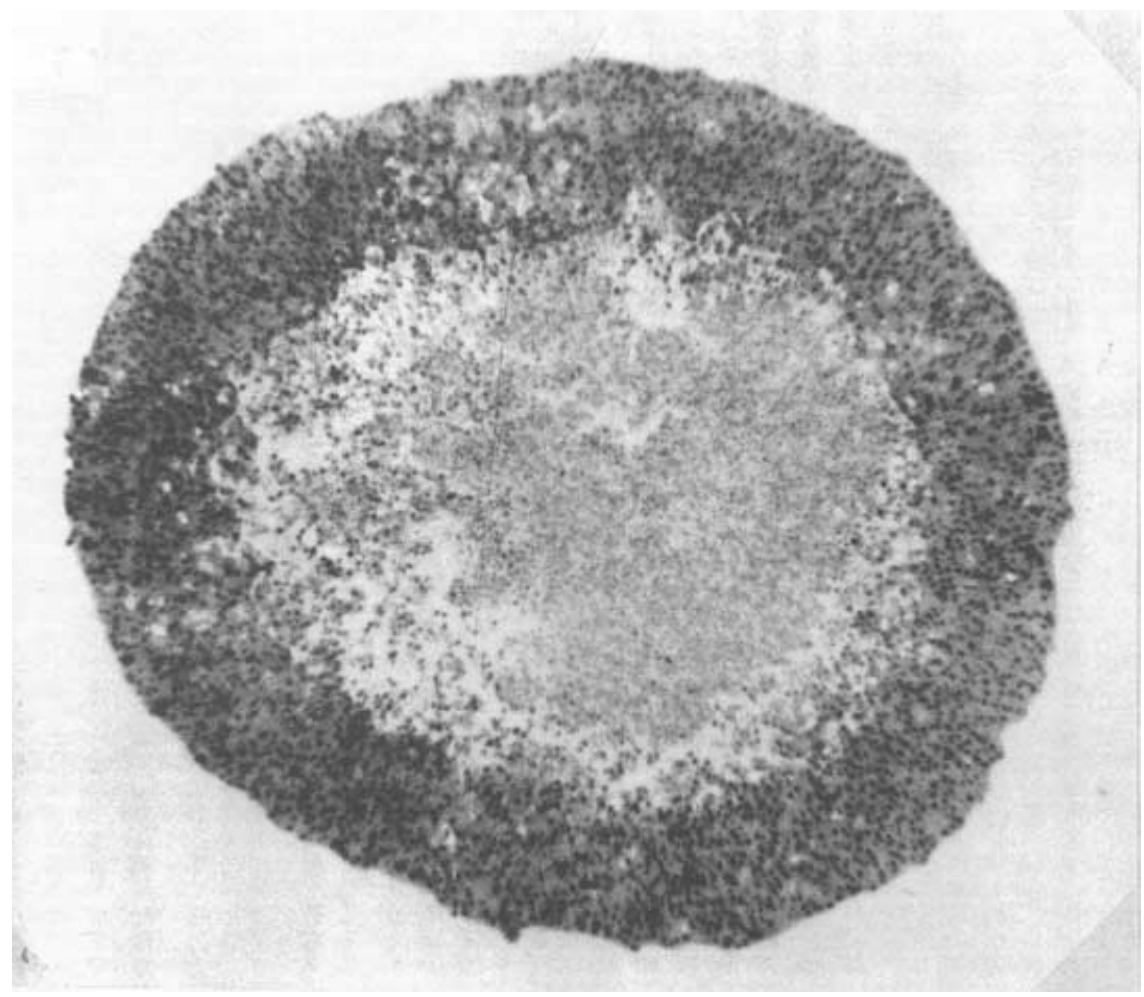

Fig. 1. An illustration of the structure of a multicellular tumour spheroid, with an outer rim of proliferating cells and an inner necrotic core; these are separated by a layer of quiescent cells. The spheroid diameter is $1.4 \mathrm{~mm}$. [Reproduced from Sutherland et al. (Cancer Res. $46,5320-5329,1986)$ by courtesy of the publisher and the authors]. 
of oxygen and glucose play an important role: experiments using microelectrodes have shown that these decrease from the outside of a spheroid to the inside (e.g. Mueller-Klieser et al., 1986). However, quiescence still develops in spheroids where oxygen and glucose levels are not low enough to prevent cell proliferation (Freyer \& Sutherland, 1986; Casciari et al., 1992), indicating the involvement of other regulators such as growth factors; details of these remain unclear. An important recent development is the finding that quiescent cells have reduced mitochondrial function (but not number) (Freyer, 1998), but again the implications of this for regulation of quiescence are not yet clear.

Mathematical modelling of avascular tumour growth has a long history, dating back to the work of Thomlinson \& Gray (1955). The majority of previous modelling falls into two categories. The simplest approach is that developed by Mueller-Klieser and coworkers, who use ODE models to predict the variation in the concentration of oxygen and other nutrients through a spheroid, using comparisons with experimental data to determine unknown quantities such as diffusion rates (e.g. Mueller-Klieser, 1984; Groebe \& Mueller-Klieser, 1996). A more sophisticated category of modelling has developed from an influential paper of Greenspan (1972), who proposed dividing the proliferating, quiescent and necrotic cells into separate compartments, with behaviour controlled by mitotic inhibitors. This reduces the problem to determining the location of the interfaces between the compartments, which are controlled by nutrient and inhibitor levels. This approach has been used widely (e.g. Shymko \& Glass, 1976; Adam, 1986; Chaplain \& Britton, 1993); recent extensions include the study of asymmetric growth (Byrne \& Chaplain, 1997) and detailed analytical studies of model solutions (Byrne, 1999; Friedman \& Reitich, 1999). In addition to these two modelling approaches, which both address spatial structure, there have been models which focus simply on the temporal evolution of cell numbers, without considering spatial structure: such models are reviewed by Bajzer et al. (1997).

Although it has proved an extremely valuable formalism, the division of proliferating, quiescent and necrotic cells into separate spatial compartments is artificial. Detailed experimental investigations based on measuring oxygen consumption rates show that the transitions between the layers can be gradual, rather than sharp (Hystad \& Rofstad, 1994). To our knowledge, the only previous modelling work on the spatial structure of avascular tumours that does not assume distinct cell layers is by Ward \& King $(1997,1999)$. Their model is formulated in terms of densities of live and dead cell populations, with quiescent cells omitted for simplicity, and they assume that the cell population is space-filling, with tumour growth due entirely to generation of new cells by division. In Ward \& King (1997), the dependence of quantities such as the viable rim thickness and growth velocity on model parameters is studied, using a series of asymptotic approximations; in the 1999 paper, the work is extended to include diffusion of "cellular material", enabling growth saturation to be studied.

The modelling work in this paper incorporates cell movement into the growth of tumour spheroids. This is not a new idea: in particular, it was the focus of the modelling work of McElwain \& Pettet (1993); however, their study did not explicitly involve separate sub-populations of cells. Since cell movement requires 
at least a small amount of extracellular space, Ward \& King's $(1997,1999)$ work neglects such movement. However, experimental studies show significant extracellular space (up to 20\%) in some multicellular spheroids (Durand, 1990), with the composition of extracellular matrix being well characterised (Nederman et al., 1984; Paulus et al., 1994); moreover, the limited available evidence suggests that avascular tumours in vivo contain more extracellular space than spheroids (Davies et al., 1997). In this paper, we propose a new model for avascular tumour growth, which does not assume sharp divisions in space between cell types, and in which tumour growth is driven by cell movement acting in concert with proliferation.

\section{Model formulation}

Since we are not assuming separate compartments with sub-populations of cells, we formulate our model in terms of cell densities, denoted $p(x, t), q(x, t)$ and $n(x, t)$ for proliferating, quiescent and necrotic cells, respectively. Here $t$ denotes time and $x$ is a spatial coordinate in a one-dimensional domain. As discussed above, we assume that the tumour is thin, approximately disc-shaped. For mathematical simplicity, we restrict attention to one spatial dimension, corresponding to a crosssection through the tumour; this facilitates analysis, and will not have a significant effect except when the tumour radius is very small.

Necrotic cells are of course non-motile, but both the proliferating and quiescent sub-populations will move, and the basic difficulty in model formulation is the way in which their interaction affects motility. We assume that cell movement is unbiased, neglecting any directed movement - inclusion of the effects of oxygen and other gradients is a natural extension (see McElwain \& Pettet, 1993). For a single cell population, unbiased movement is traditionally modelled by linear diffusion, with a long history of successful application to a variety of cell biology problems (e.g. Keller \& Segel, 1971; Sherratt \& Murray, 1990; Chaplain \& Stuart, 1993). However, for interacting populations, there is no established modelling formalism. Linear diffusion of each cell type would imply mixing of two adjacent populations, which is inappropriate for the close-packed cell populations involved in early tumour growth, since the presence of a cell of one type limits the movement of a cell of the other type from moving. This phenomenon is known as "contact inhibition of migration" (Abercrombie et al., 1970), and is very well established in a wide range of cell types. Contact inhibition will not prevent a tumour from growing of course - its proliferative advantage over normal tissue ensures this - but it will have a significant effect on the rate of growth.

We therefore use a new cell movement term, which reflects this contact inhibition of migration in a simple way. We assume that the overall cell flux (of both proliferating and quiescent populations) can be taken as $-\frac{\partial}{\partial x}(p+q)$, corresponding to linear diffusion; by a suitable rescaling of space, any motility coefficient can be taken as 1 . We assume that the two cell populations have equal motility, that is unaffected by necrotic cells. Thus, of the total cell flux, a fraction $p /(p+q)$ will be proliferating cells, giving the flux $-[p /(p+q)] \frac{\partial}{\partial x}(p+q)$, with the flux of quiescent cells given similarly. These expressions give new motility terms which, although very simple, reflect the key property of contact inhibition. The terms have been 
studied previously, in the context of a very simple competition model, by Sherratt (2000); here we consider them in the more specific context of the development of structure within an avascular tumour.

We assume that proliferating cells divide at a rate that is limited by crowding effects of the total cell population, and that proliferating cells become quiescent at a rate that depends on the concentration $c(x, t)$ of a generic nutrient/growth factor. Similarly, the quiescent cells become necrotic at a rate that depends on $c$. This gives the model equations

$$
\begin{aligned}
& \frac{\partial p}{\partial t}=\frac{\partial}{\partial x}\left[\frac{p}{p+q} \frac{\partial}{\partial x}(p+q)\right]+g(c) p(1-p-q-n)-f(c) p \\
& \frac{\partial q}{\partial t}=\frac{\partial}{\partial x}\left[\frac{q}{p+q} \frac{\partial}{\partial x}(p+q)\right]+f(c) p-h(c) q \\
& \frac{\partial n}{\partial t}=h(c) q .
\end{aligned}
$$

Here, we are assuming that cell populations have been rescaled so that a value of 1 corresponds to a completely close-packed population; we also fix $g(0)=1$ by a suitable rescaling of time. The functions $f($.$) and h($.$) will be decreasing with$ $f(+\infty)=h(+\infty)=0$, and $g($.) will be increasing. Moreover we expect that the rate (per cell) of entry into quiescence to be larger than the rate of necrosis, at any given nutrient/growth factor level, so that $f(c)>h(c)$. However the functional forms cannot be specified in further detail because of the uncertainty surrounding the transition between the cell types (discussed in $\S 1$ ).

To determine the appropriate equation for $c(x, t)$, we must consider the context in which the tumour is growing. The majority of previous models for avascular tumour growth apply specifically to multicellular spheroids in vitro. However, we focus attention on avascular tumours in vivo, which will differ from the in vitro case in two main regards. Firstly, the tumour will not be growing in a medium of nutrient-rich fluid. In particular, since the vast majority of primary tumours develop in the epithelia of tissues (these are known as carcinomas and represent about $80 \%$ of clinically observed cancers), they will usually be surrounded by a dense cell population. This will significantly retard growth, to an extent that can be estimated via mathematical modelling (Sherratt, 2000), but we do not anticipate that it will significantly alter the internal tumour structure, and thus we neglect this effect initially, for simplicity - we consider the effects of surrounding tissue in $\S 4$. Note that for secondary tumours, a very wide range of environments is possible: for instance, the tumour may develop immediately adjacent to a blood vessel from which it has entered the tissue, a case considered specifically in recent modelling by Please et al. (1999).

Secondly, an in vivo tumour will differ from a multicellular spheroid in not necessarily being spherical. In fact, because they initially develop in epithelia, one expects that many primary tumours initially form as mainly two-dimensional structures, growing within the epithelium before expanding into surrounding tissues. There is very little data on this, because inevitably most in vivo tumours are not observed until they are relatively large. Since the case of spherical avascular 
tumours has been studied in such detail previously, we restrict attention here to the two-dimensional case. This significantly affects the equation for $c$, because in a spherical tumour, nutrient can only reach the centre by diffusing through the whole structure, while in a structure that is approximately two-dimensional, nutrient can access the whole tumour much more readily, from underlying tissue. Thus, we use the following model equation for our generic nutrient $c(x, t)$ :

$$
\frac{\partial c}{\partial t}=D_{c} \frac{\partial^{2} c}{\partial x^{2}}+k_{1} c_{0}[1-\alpha(p+q+n)]-k_{1} c-k_{2} p c .
$$

Here, the access of nutrient from underlying tissue is represented by the source term $k_{1} c_{0}[1-\alpha(p+q+n)]$. Thus we assume that the effectiveness of this source term decreases with overall cell density; the parameter $\alpha \in(0,1]$, and $c_{0}$ is the nutrient concentration in the absence of a tumour cell population.

Numerical simulations of this model $(1,2)$ demonstrate the familiar structure of proliferating rim and a band of quiescent cells around a growing necrotic core (Figure 2a). Parameter values and the functional forms of $f(),. g($.$) and h($.) will of course affect the width and cell density of the regions, as well as the overall speed of tumour growth, and this will be discussed further in $\S 3$. Moreover, if $f(c)$ decreases to zero at a finite value of $c$, then the solution can have a quite different qualitative form, with a growing mass of entirely proliferating cells (Figure 2b). Numerical experiments indicate that this occurs when the parameter $\alpha$ is fairly small, so that nutrient supply to the tumour from underlying tissue is large enough to prevent cells from entering quiescence. For other parameter values, solutions can also consist of a proliferating rim around a wholly or partially quiescent core (not illustrated for brevity). The possibility of a threshold level of nutrient above which cells do not become quiescent is entirely realistic. Thus an important goal for analysis is the determination of criteria for the occurrence of the new types of solutions, such as that illustrated in Figure $2 b$, that are introduced by our assumption of nutrient supply from underlying tissue.

\section{Reduced model}

In this section we consider a reduced model that focusses on the cell dynamics within the tumour, given by assuming that the nutrient kinetics are at a quasi-steady state. This simplification, which greatly facilitates analysis, gives the equations

$$
\begin{aligned}
\frac{\partial p}{\partial t} & =\frac{\partial}{\partial x}\left[\frac{p}{p+q} \frac{\partial}{\partial x}(p+q)\right]+g(c) p(1-p-q-n)-f(c) p \\
\frac{\partial q}{\partial t} & =\frac{\partial}{\partial x}\left[\frac{q}{p+q} \frac{\partial}{\partial x}(p+q)\right]+f(c) p-h(c) q \\
\frac{\partial n}{\partial t} & =h(c) q \\
c & =c_{0} \gamma[1-\alpha(p+q+n)] /(\gamma+p)
\end{aligned}
$$


(a)

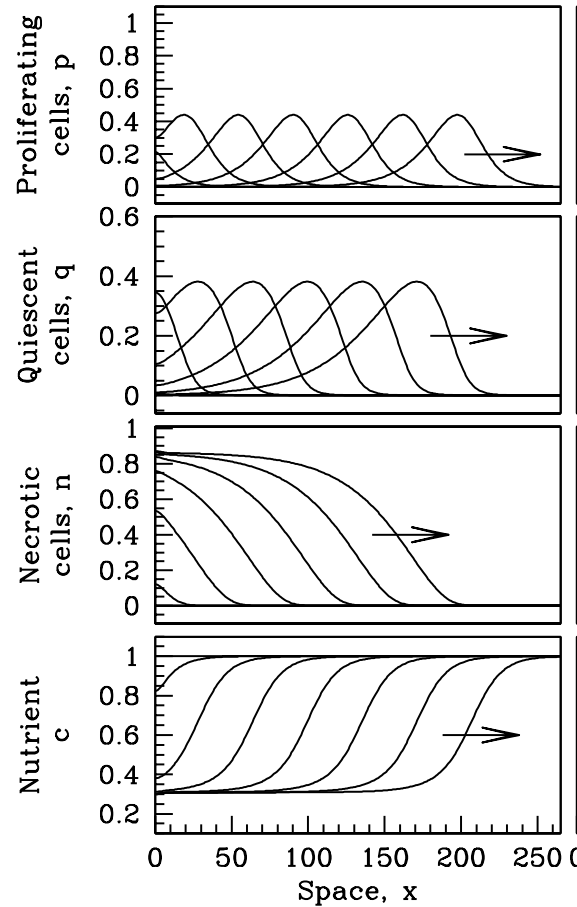

(b)
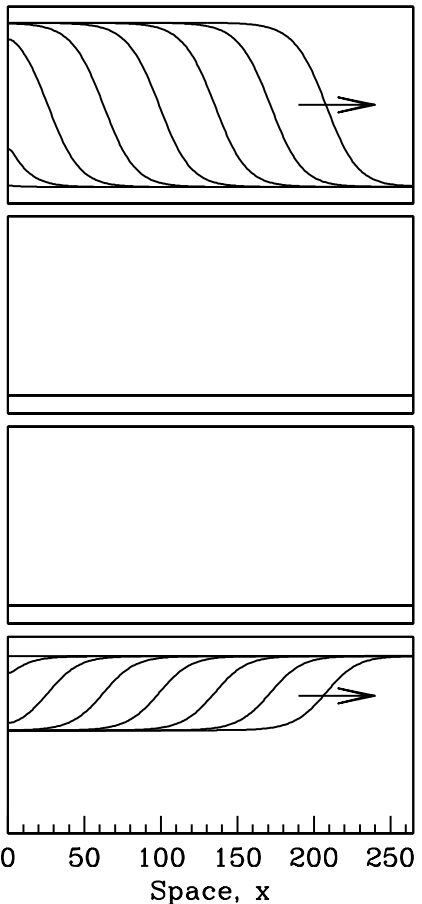

Fig. 2. Numerical solutions of the model equations $(1,2)$. (a) A solution with the form of an advancing pulse of proliferating cells $(p)$, with a band of quiescent cells $(q)$ and a necrotic core $(n)$ behind this; in parallel with this is a receding wave of nutrient $(c)$. (b) A solution of a different qualitative form, with an advancing front of proliferating cells $(p)$ and a receding wave of nutrient $(c)$, with no quiescent or necrotic cells. This corresponds to the nutrient supply from surrounding tissue being sufficient to keep all the tumour cells proliferating, with no entry into quiescence. In both cases, the solutions are plotted as a function of space at times $t=0,3,6, \ldots, 21$, with arrows indicating increasing time. The parameter values are $k_{1}=8, k_{2}=1, D_{c}=10, c_{0}=1$, and (a) $\alpha=0.8$, (b) $\alpha=0.3$. The function $g(c)=1+0.2 c$ and $h(c)=\frac{1}{2} f(c)$, with (a) $f(c)=\frac{1}{2}[1-\tanh (4 c-2)]$, (b) $f(c)$ is a step function defined by $f(c)=1$ if $c<0.5$ and $f(c)=0$ for $c>0.5$. The initial conditions were $q \equiv n \equiv 0$, $c \equiv 1, p=0.01 \exp (-0.1 x)$, and the boundary conditions used at $x=0$ and $x=265$ were $p_{x}=q_{x}=c_{x}=0$ (no boundary conditions are required for $n$ ). At $x=0$, this represents symmetry, but the boundary condition at $x=265$ is an artificial one, required because we cannot solve numerically on a semi-infinite domain; it is not significant since we stop the solution before the wave of proliferating cells reaches this boundary.

where $\gamma=k_{1} / k_{2}$. A typical solution of this reduced model is illustrated in Figure 3, showing advancing pulses of proliferating and quiescent cells, with a growing necrotic core behind this; equation (3c) implies a receding wave of nutrient (not illustrated).

Our objective in this section is to obtain an analytical approximation to these wave solutions, and thus we consider solutions of travelling wave form, $p(x, t)=$ 


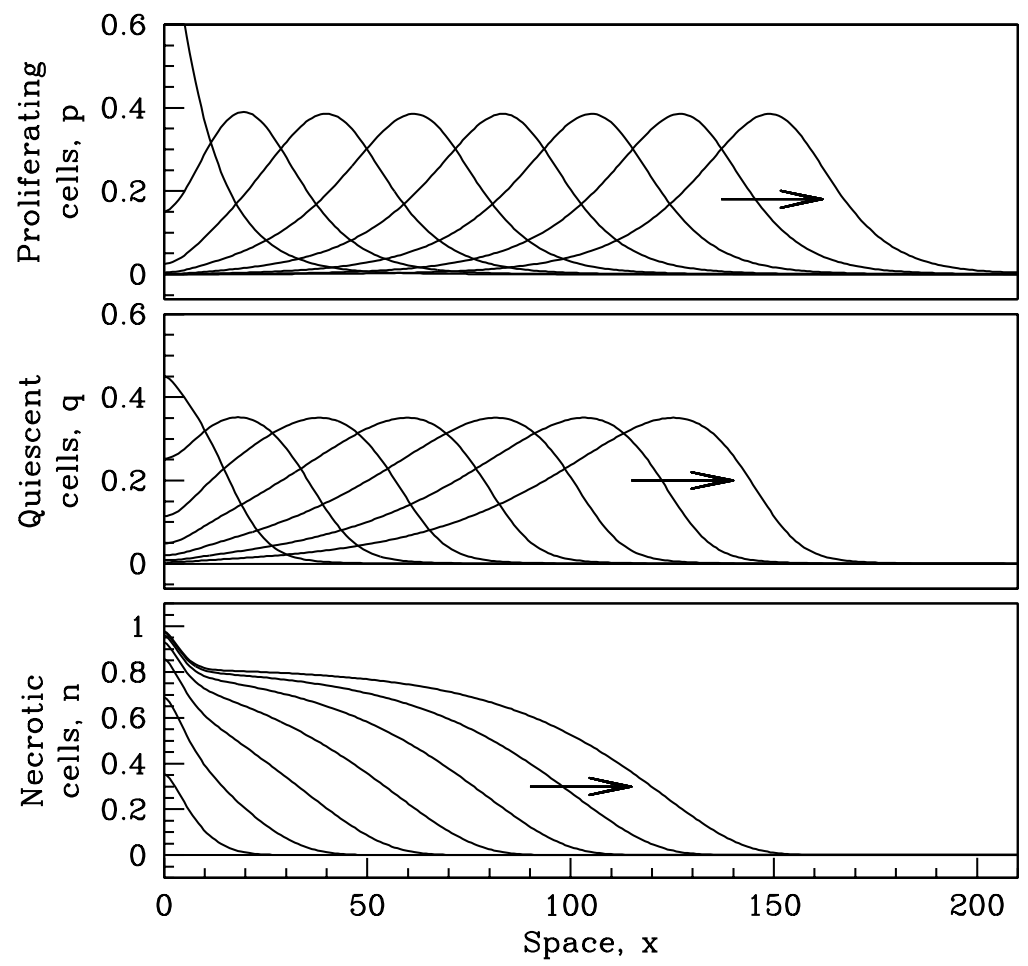

Fig. 3. A numerical solution of the reduced model (3), showing an advancing pulse of proliferating cells $(p)$, with a band of quiescent cells $(q)$ and a necrotic core $(n)$ behind this. The solution is plotted as a function of space at times $t=0,2,4, \ldots, 14$, with arrows indicating increasing time. The parameter values are $\gamma=10, c_{0}=1$ and $\alpha=0.9$, with $f(c)=\frac{1}{2}[1-\tanh (4 c-2)], g(c)=1+0.1 c$, and $h(c)=\frac{1}{2} f(c)$. The initial conditions were $q \equiv n \equiv 0, p=\exp (-0.1 x)$, and the boundary conditions used at $x=0$ and $x=210$ were $p_{x}=q_{x}=0$ (no boundary conditions are required for $n$ ). At $x=0$, this represents symmetry, but as in Figure 3, the boundary condition at $x=210$ is an artificial one, required because we cannot solve numerically on a semi-infinite domain.

$P(z), q(x, t)=Q(z), n(x, t)=N(z), z=x-a t$, where $a$ is the wave speed. Substituting these solution forms into (3) gives the ODEs

$$
\begin{array}{r}
\left(\frac{P\left(P^{\prime}+Q^{\prime}\right)}{P+Q}\right)^{\prime}+a P^{\prime}+g(C) P(1-P-Q-N)-f(C) P=0 \\
\left(\frac{Q\left(P^{\prime}+Q^{\prime}\right)}{P+Q}\right)^{\prime}+a Q^{\prime}+f(C) P-h(C) Q=0 \\
a N^{\prime}+h(C) Q=0 \\
\text { where } C=c_{0} \gamma[1-\alpha(P+Q+N)] /(\gamma+P)
\end{array}
$$




\subsection{The wave speed}

We begin by considering the speed $a$ of the travelling wave solutions satisfying (4). Linearising (4) ahead of the wave (about $P=Q=0$ ) and assuming $P(z)=$ $\hat{P} \exp (-\xi z), Q(z)=\hat{Q} \exp (-\xi z), N(z)=\hat{N} \exp (-\xi z)$ to leading order, gives

$$
\begin{aligned}
\xi^{2} \hat{P}-a \xi \hat{P}+\left[g\left(c_{0}\right)-f\left(c_{0}\right)\right] \hat{P} & =0 \\
\xi^{2} \hat{Q}-a \xi \hat{Q}+f\left(c_{0}\right) \hat{P} & =0 \\
-a \xi \hat{N}+h\left(c_{0}\right) \hat{Q} & =0
\end{aligned}
$$

Thus for nontrivial solutions, we must have

$$
\xi=\frac{1}{2}\left(a \pm \sqrt{a^{2}-4\left[g\left(c_{0}\right)-f\left(c_{0}\right)\right]}\right)
$$

Since $P, Q$ and $N$ must be positive, we require $\xi$ to be real, so that $a \geq 2$ $\sqrt{g\left(c_{0}\right)-f\left(c_{0}\right)}$. This existence of a minimum possible wave speed, derived by linearising ahead of the wave, is familiar from scalar reaction-diffusion equations (Fisher, 1937; Kolmogoroff et al., 1937). In these cases it is known that a wave moving with the minimum possible speed evolves from sufficiently localised initial data; this is the initial data that is relevant in most biological applications, including tumour growth. Thus we anticipate that the speed of tumour growth implied by (3) will be $2 \sqrt{g\left(c_{0}\right)-f\left(c_{0}\right)}$, and this is confirmed by numerical simulations.

\subsection{Approximate travelling wave equations: $f(c)>0$ for all $c$}

Although the wave speed is a key feature of the solutions of (3), the shape of the waves is also of particular importance - for instance, it is important to know how the width and density of the bands of proliferating and quiescent cells depends on model parameters. To study this, we adapt a method developed by Canosa (1973) for approximate solution of the Fisher equation. This involves rescaling the travelling wave coordinate by writing $\zeta=-z / a$, giving

$$
\begin{array}{r}
\frac{1}{a^{2}} \frac{d}{d \zeta}\left(\frac{P \frac{d}{d \zeta}(P+Q)}{P+Q}\right)-\frac{d P}{d \zeta}+g(C) P(1-P-Q-N)-f(C) P=0 \\
\frac{1}{a^{2}} \frac{d}{d \zeta}\left(\frac{Q \frac{d}{d \zeta}(P+Q) / d \zeta}{P+Q}\right)-\frac{d Q}{d \zeta}+f(C) P-h(C) Q=0 \\
\frac{d N}{d \zeta}-h(C) Q=0
\end{array}
$$

We have shown above that $a \geq 2 \sqrt{g\left(c_{0}\right)-f\left(c_{0}\right)}$. Now $g\left(c_{0}\right) \geq g(0)=1$, and we expect $f\left(c_{0}\right)$ to be very small, since there will be little or no entry into quiescence when $c=c_{0}$, the nutrient level present away from the tumour. Thus $1 / a^{2}$ 
will be small; neglecting terms containing this factor gives two coupled first order equations, which are most conveniently rewritten as

$$
\begin{aligned}
d P / d \zeta & =g(C) P(1-M)-f(C) P \\
d M / d \zeta & =g(C) P(1-M) \\
\text { where } \quad C & =c_{0} \gamma(1-\alpha M) /(\gamma+P) .
\end{aligned}
$$

Here $M \equiv P+Q+N$. For notational convenience in the following, we define $\phi(C) \equiv f(C) / g(C)$.

We are looking for a solution of (6) with $P=M=0$ at $\zeta=-\infty$ (corresponding to $x=+\infty$ : ahead of the wave). We begin by considering the case of $\phi(C)>0$ for all finite $C$; then all equilibrium points must have $P=0$, so that $P=0, M>0$ at $\zeta=+\infty$ (behind the wave). In this subsection, we will study the solution form in this case, using phase plane arguments.

Straightforward linear analysis shows that $P=M=0$ is an unstable steady state, with the unstable eigenvector having slope $1-\phi\left(c_{0}\right)$. (The other eigenvector has slope zero, corresponding to neutral stability along the $M$-axis). Therefore a travelling wave solution must correspond to the unique trajectory leaving $(0,0)$ along this eigenvector. Explicit differentation shows that

$$
\frac{d^{2} P}{d M^{2}}=\frac{-\phi(C)}{(1-M)^{2}}+\frac{\phi^{\prime}(C)}{(1-M)(\gamma+P)}\left[\alpha \gamma+C \frac{d P}{d M}\right] .
$$

Since $f($.$) and g($.$) are decreasing and increasing functions respectively, \phi(.) \equiv$ $f(.) / g($.$) is decreasing. Thus d P / d M$ decreases with $M$ whenever it is positive (i.e. while a trajectory is increasing, it is concave down). Thus the trajectory leaving $(0,0)$ remains below the line $P=\left[1-\phi\left(c_{0}\right)\right] M$.

Consider now the $P$ nullcline, $\phi(C)=1-M$. Using (6c), this has the equation

$$
P=\gamma\left[\frac{1-\alpha M}{\phi^{-1}(1-M) / c_{0}}-1\right] \text {. }
$$

Provided $\phi(c)$ remains strictly positive for finite $c$, the function $\phi^{-1}($.$) is decreas-$ ing, with $\phi^{-1}(0)=+\infty$ (because $f(+\infty)=0$ ). Thus the $P$ nullcline decreases monotonically in the $M<1$ portion of the $P-M$ plane, from $+\infty$ at $M=1-\phi(0)$ (this can be positive or negative) to a negative value at $M=1$, as illustrated in Figure 4; note that behaviour for $M>1$ is not relevant. We have shown already that a trajectory leaving $(0,0)$ must remain below $P=\left[1-\phi\left(c_{0}\right)\right] M$; moreover the trajectory must have positive slope until it intersects the $P$ nullcline. Therefore this intersection must occur (see Figure 4).

Beyond this point of intersection with the $P$ nullcline, the trajectory has negative slope, and cannot intersect the nullcline again since this nullcline is a decreasing function of $M$, and at such an intersection point, the slope of the trajectory would have to be zero. Moreover, the trajectory must remain in the region $M<1$, since $d M / d z=0$ at $M=1$. Therefore the trajectory must terminate on the $M$ axis, at a point between $M=1$ and the intersection of the $P$ nullcline with the $M$ axis. Thus this trajectory has the required form for a travelling wave solution. 


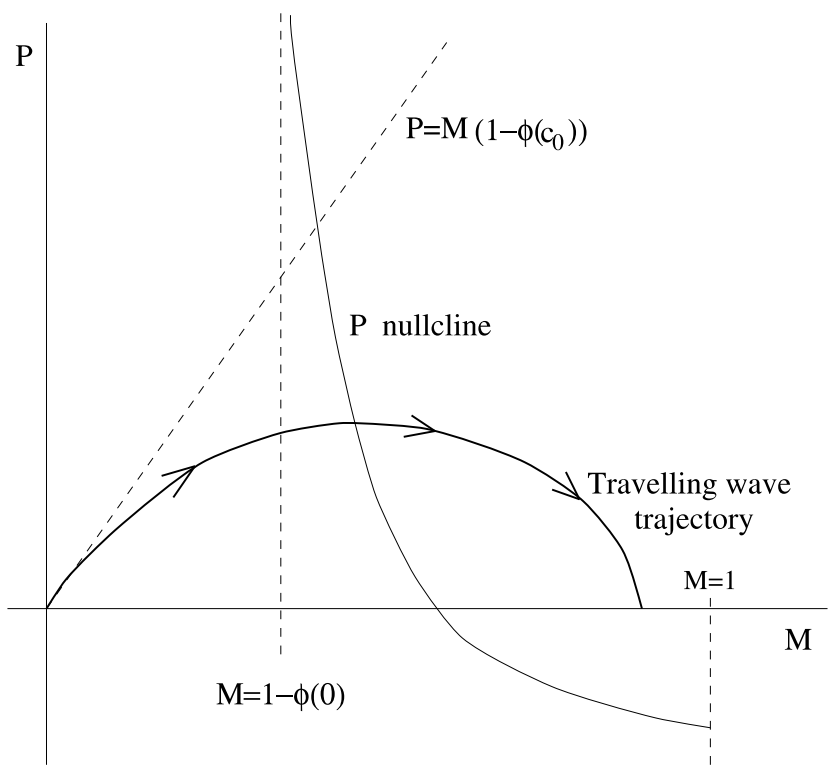

Fig. 4. An illustration of the form of the travelling wave trajectory in the $P-M$ phase plane, when $f(c)>0$ for all finite $c$. The trajectory lies below the line $P=\left(1-\phi\left(c_{0}\right)\right) M$, and thus must intersect the $P$ nullcline, at a point corresponding to the peak of the pulse wave of $P$. The trajectory then decreases, terminating on the $M$-axis.

From the wave trajectory illustrated in Figure 3, the solution forms for $Q(\zeta)$ and $N(\zeta)$ can easily be deduced, via $Q=M-P-N$ and $d N / d \zeta=h(C) \cdot(M-P-N)$. Provided that $h(C)>0$ for all finite $C, Q \rightarrow 0$ as $\zeta \rightarrow+\infty$, corresponding to a band of quiescent cells, with $N(+\infty)>0$, representing a necrotic core. This is the standard structure for an avascular tumour, familiar from multicell spheroids. However, if $h(c)$ decreases to zero at a finite value of $c$ that is greater than $C(+\infty)=c_{0}(1-\alpha)$, necrosis will not be complete, with the core of the tumour consisting of a mixture of necrotic and quiescent cells.

\subsection{Approximate travelling wave equations: $f(c)=0$ for sufficiently large $c$}

If $\phi(c)$ decreases to zero at a finite value of $c$, say $c=c^{*}$ (with $c^{*}<c_{0}$ ), an important new feature is introduced, namely that there are equilibrium points of (6) with $P>0$. Specifically, all points with $M=1$ and $C \geq c^{*}$ (i.e. $P \leq$ $\left.\gamma\left(c_{0}-c^{*}-c_{0} \alpha\right) / c^{*}\right)$ are equilibria, in addition to points with $P=0$. For $\alpha$ sufficiently large (including in particular the case $\alpha=1$ ) this does not alter the qualitative behaviour discussed in $\$ 3.2$. However, for smaller $\alpha$ there can be a travelling wave trajectory terminating with $M=1$ and either $P<1$ or $P=1$. Recalling that $M \equiv P+Q+N$, these cases correspond respectively to $Q, N>0$ (a mixture of proliferating, quiescent and necrotic cells throughout the tumour) or $Q=N=0$ (no cells entering quiescence); intuitively, these occur for small $\alpha$ since this corresponds to a large influx of nutrient into the tumour from surrounding 
tissue. We have not attempted to classify the possible behaviours in general; rather, we do so in the particular simple case of piecewise constant $f($.$) and constant g($.$) ,$ for which exact travelling wave solutions can be determined.

\subsection{Analytical approximation for piecewise constant $f($.}

In general, we have not been able to find an exact solution for the travelling wave trajectory in (4). However, such a solution can be found in the special case of $g(c) \equiv 1$ with $f(c)$ a step function, defined by:

$$
f(c)= \begin{cases}0, & c>c^{*} \\ 1, & c<c^{*}\end{cases}
$$

where $c^{*} \in\left(0, c_{0}\right)$ is a positive constant. Although this is a rather degenerate case, its solution gives valuable insights into the travelling wave form. We will show that the solution can have four different qualitative forms, depending on parameter values. Equation (6) implies

$$
\frac{d P}{d M}=1-\frac{\lambda}{1-M} \Rightarrow P=M+\lambda \log (1-M)+K
$$

Here $K$ is a constant of integration, and $\lambda$ is given by

$$
\lambda= \begin{cases}0, & c_{0} \alpha \gamma M+c^{*} P<\gamma\left(c_{0}-c^{*}\right) \\ 1, & c_{0} \alpha \gamma M+c^{*} P>\gamma\left(c_{0}-c^{*}\right) .\end{cases}
$$

Henceforth, we denote by $\mathcal{L}$ the line in the $M-P$ plane given by $c_{0} \alpha \gamma M+c^{*} P=$ $\gamma\left(c_{0}-c^{*}\right)$; this is the line along which $C=c^{*}$.

For $\zeta$ sufficiently large and negative, $\lambda=0$ and we have the solution $P=M$, with $K=0$ because the trajectory originates from $(0,0)$. This solution will apply until the trajectory intersects the line $\mathcal{L}$, at the point

$$
P=M=\gamma\left(c_{0}-c^{*}\right) /\left(c_{0} \alpha \gamma+c^{*}\right) \equiv \psi, \text { say }
$$

For some parameter values, $\psi>1$. In this case, which we refer to as case A, $P=M$ for the entire solution, with the trajectory terminating at $(1,1)$, and with $C>c^{*}$ throughout (Figure 5a); this corresponds to nutrient supply within the tumour being great enough to prevent any cell quiescence, as discussed above.

If $\psi<1$, then beyond the point at which the trajectory and $\mathcal{L}$ intersect, the possible solution having $\lambda=1$ is

$$
P=M+\log (1-M)-\log \left(1-\frac{\gamma\left(1-c^{*} / c_{0}\right)}{\alpha \gamma+c^{*} / c_{0}}\right) \equiv \mathcal{H}(M), \quad \text { say } .
$$

Provided that $\mathcal{H}^{\prime}(\psi)$ is greater than the slope of $\mathcal{L}$, namely $-c_{0} \alpha \gamma / c^{*}$, the trajectory will continue along $P=\mathcal{H}(M)$. The function $\mathcal{H}(M)$ decreases monotonically in $(0,1)$, with a unique zero in this interval, at $M=M_{0}$, say, and the subsequent behaviour depends on the value of $M_{0}$. If $M_{0}>\left(1-c^{*} / c_{0}\right) / \alpha$, then the curve $P=\mathcal{H}(M)$ intersects the $M$ axis before the line $\mathcal{L}$, and thus the trajectory consists simply of this curve together with the line $P=M$ (case D, illustrated in Figure 5d). 

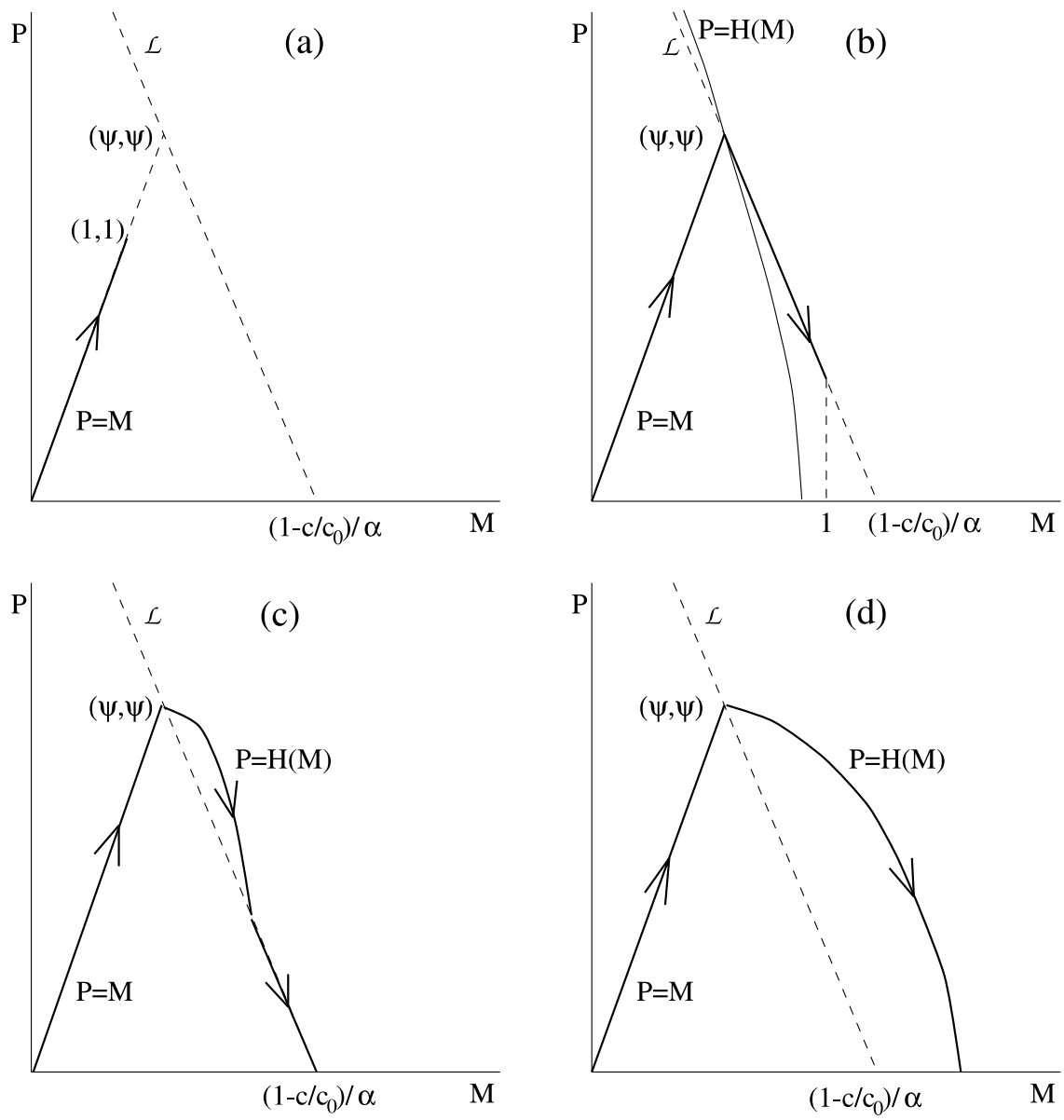

Fig. 5. An illustration of the possible forms of the travelling wave trajectory in the $P-M$ phase plane, when $f($.$) is the step function (7). Parts (a), (b), (c) and (d) of the figure denote$ cases $\mathrm{A}, \mathrm{B}, \mathrm{C}$ and $\mathrm{D}$, as described in the main text.

However, if $M_{0}<\left(1-c^{*} / c_{0}\right) / \alpha$, then the intersection with the line $\mathcal{L}$ occurs first (case $\mathrm{C}$, illustrated in Figure 5c): recall that the line $\mathcal{L}$ corresponds to $C=c^{*}$ and to a switch in the value of $\lambda$. Beyond this intersection, if the solution were to lie below the line $\mathcal{L}$, then the solution would have $d P / d M>0$, while if it were above $\mathcal{L}$, it would satisfy $P-M-\log (1-M)=$ constant. Each of these solutions drives the trajectory towards $\mathcal{L}$, and thus the remainder of the solution must be given simply by the line $\mathcal{L}$ itself (Figure 5c). Note that in cases $\mathrm{C}$ and $\mathrm{D}$, the trajectory reaches the $M$-axis at a value of $M$ that is $<1$ (shown below).

The remaining case to consider (case B) is when $\mathcal{H}^{\prime}(\psi)<-c_{0} \alpha \gamma / c^{*}$. Then the curve $P=\mathcal{H}(M)$ enters the region $C>c^{*}$ as $M$ increases from $M=\psi$, and is thus not a possible trajectory. In this case, the trajectory again lies along the line $\mathcal{L}$ (Figure $5 \mathrm{~b}$ ), since solutions on either side of the line would drive the solution 
towards $\mathcal{L}$. Whenever this case occurs, the line $\mathcal{L}$ intersects the $M$ axis at a value of $M>1$ (shown below), and thus the trajectory terminates at $M=1$ with $P>0$. This corresponds to nutrient supply within the tumour being sufficient to maintain a mixture of proliferating and quiescent cells.

The distinctions between these four cases can easily be made explicit in terms of parameters. The arguments above show that the conditions for the four possible solution forms are as follows:

Case A: $\psi>1$

Case B: $\quad \psi<1$ and $\mathcal{H}^{\prime}(\psi)<-c_{0} \alpha \gamma / c^{*}$

Case C: $\quad \psi<1$ and $\mathcal{H}^{\prime}(\psi)>-c_{0} \alpha \gamma / c^{*}$ and $1-c^{*} / c_{0}>\alpha$ or $\mathcal{H}\left(\frac{1-c^{*} / c_{0}}{\alpha}\right)<0$

Case D: $\quad \psi<1$ and $\mathcal{H}^{\prime}(\psi)>-c_{0} \alpha \gamma / c^{*}$ and $1-c^{*} / c_{0}<\alpha$ and $\mathcal{H}\left(\frac{1-c^{*} / c_{0}}{\alpha}\right)>0$.

In distinguishing between cases $\mathrm{C}$ and $\mathrm{D}$, the possibility that $\left(1-c^{*} / c_{0}\right)>\alpha$ must be treated separately because $\mathcal{H}(M)$ is only defined for $M<1$. Straightforward algebraic manipulation shows that the above conditions simplify to the following:

Case A: $\quad \alpha<1-c^{*} / c_{0}-c^{*} /\left(c_{0} \gamma\right)$

Case B: $\quad \alpha \in\left(1-c^{*} / c_{0}-c^{*} /\left(c_{0} \gamma\right), 1-c^{*} / c_{0}\right)$

Case C: $\quad \alpha \in\left(1-c^{*} / c_{0}, \alpha_{c r i t}\right)$

Case D: $\quad \alpha>\alpha_{\text {crit }}$

where $\alpha_{\text {crit }}$ is given by

$$
c_{0} \alpha_{c r i t}\left[c_{0} \gamma \alpha_{c r i t}-\gamma\left(c_{0}-c^{*}\right)+c^{*}\right]=\frac{c^{*}\left(c_{0}-c^{*}\right)}{1-\exp \left\{-\left(1-c^{*} / c_{0}\right) / \alpha_{c r i t}\right\}} .
$$

We show in the Appendix that this equation has a unique positive solution for $\alpha_{\text {crit }}$. Note that the value of $\alpha_{c r i t}$ is always greater than $\left(1-c^{*} / c_{0}\right)$, but can be greater than 1 (for very small $c^{*}$ and $\gamma$ ); this would mean that case D does not arise. Note also that the critical value $1-c^{*} / c_{0}-c^{*} /\left(c_{0} \gamma\right)$ can be positive or negative. The condition $\alpha>1-c^{*} / c_{0}$ is exactly the condition that the line $\mathcal{L}$ intersects the $M$-axis at a value of $M$ that is less than 1, so that the trajectory terminates with $P=0$ in cases $\mathrm{C}$ and $\mathrm{D}$, but with $P>0$ in case $\mathrm{B}$.

As discussed in $\S 3.2$, the solutions for $Q$ and $N$ can easily be deduced from those for $P$ and $M$. Since we require $h(c)<f(c)$, we must have $h(c) \equiv 0$ for all $c \geq c^{* *}$, a critical value that is less than $c^{*}$. Thus if the $P-M$ solution implies $C \geq c^{*}$ (cases $\mathrm{A}$ and $\mathrm{B}$ ), the cells never enter necrosis. In case $\mathrm{C}$, some cells may become necrotic, depending on $c^{* *}$, but since $C(\zeta=+\infty)=c^{*}$, there is no necrotic core, but rather a core of quiescent cells that may also include some necrotic cells. Only in case D can a necrotic core develop, provided $c^{* *}>C(\zeta=+\infty)=c_{0}(1-\alpha)$. 
The solutions for $P, Q$ and $N$ as a function of $z$ in the four cases are illustrated in Figure 6. If $\alpha$ is sufficiently small, nutrient supply within the tumour is high enough to maintain a proliferating cell population (cases $\mathrm{A}$ and $\mathrm{B}$ ), with no cells entering quiescence if $\alpha$ is very small (case A). If $\alpha$ is large enough that a proliferating population cannot be sustained, but is less than $\alpha_{\text {crit }}$, then the solution

(a)

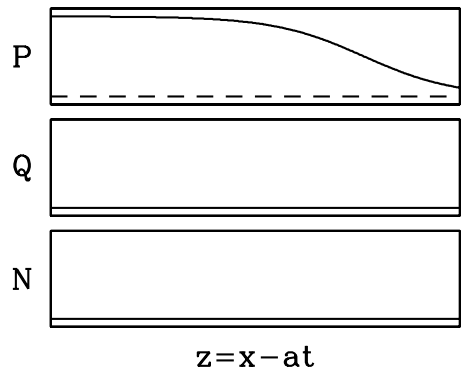

(c)

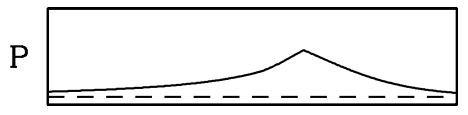

Q
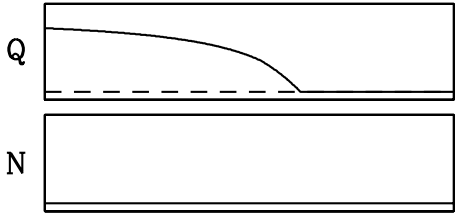

$\mathrm{z}=\mathrm{x}-\mathrm{at}$ (b)

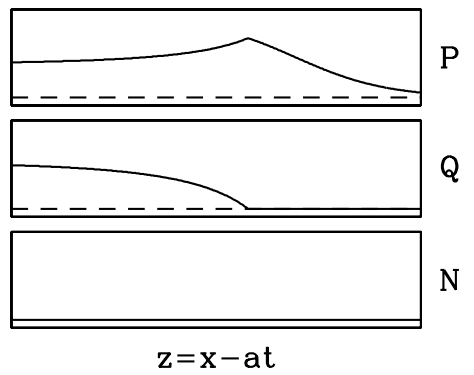

(d)

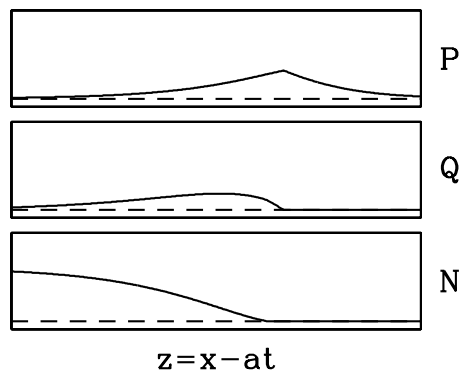

Fig. 6. An illustration of the travelling wave solution forms for $P, Q$ and $N$ in the four cases A-D, illustrated in parts (a)-(d) of the figure. The solutions are plotted as a function of $z=x-a t$ rather than $\zeta$ for ease of interpretation; recall that the calculations in $\$ 3.2$ and $\S 3.3$ are done using $\zeta=-z / a$ as independent variable. (The wave speed $a=2$ in this case). In (a), the tumour cells are all proliferating due to high nutrient input from underlying tissue. As this input is reduced, some (b) and then all (c) of the cells enter quiescence. Finally, when nutrient input from underlying tissue is low, the quiescent cells enter necrosis, and the solution corresponds to the well-known structure of bands of proliferating and quiescent cells around a necrotic core. There are two possible variations on the qualitative forms of the solutions illustrated. In case $\mathrm{C}$, some of the quiescent cells may enter necrosis, giving a core containing a mixture of quiescent and necrotic cells. Secondly, in case D, the necrotic core only develops if $\alpha>1-c^{* *} / c_{0}$; otherwise, the behaviour is as in case $\mathrm{C}$. The solutions plotted are obtained by numerical solution of the ODEs (6), with $g(c) \equiv 1$ and $f(c)$ defined by (7); $Q$ and $N$ calculated using $Q=M-P-N$ and $d N / d \zeta=h(C) \cdot(M-N-P)$. The dashed line is at the level zero in every plot: when it is not visible, this indicates that the solution is identically zero. The limits on the $P, Q$ and $N$ axes are -0.1 and 1.1 in each case, and the $z$-interval plotted is $-9<z<9$. The parameter values are $\gamma=2.5, c_{0}=1$ and $c^{*}=0.6$, which imply $\alpha_{\text {crit }} \approx 0.507$, with $\alpha$ having the value (a) 0.1 , (b) 0.3 , (c) 0.45 , (d) 0.9. The function $h(c)$ is a step function, with $h(c)=0$ for $c>0.55$ and $h(c)=0.9$ for $c<0.55$ : thus $c^{* *}=0.55$. 
consists of a rim of proliferating cells ahead of a core of quiescent cells (case C, and case $\mathrm{D}$ with $\left.\alpha<1-c^{* *} / c_{0}\right)$. Finally, in case $\mathrm{D}\left(\alpha>\alpha_{\text {crit }}\right)$ with $\alpha$ also above $1-c^{* *} / c_{0}$, the solution has the characteristic form of a proliferating rim, a band of quiescent cells, and a necrotic core.

Figure 7 shows the comparison between the analytical structure described above, and numerical solutions of the reduced model (3), for $g(c) \equiv 1$, but with $f(c)$ a switching function that is smooth but steep. Comparison with Figure 5 shows that the analytical form we have derived for step function $f($.$) provides a good ap-$ proximation to this case. Our analysis can be used to predict the dependence on parameter values of key aspects of the model solution, as follows.

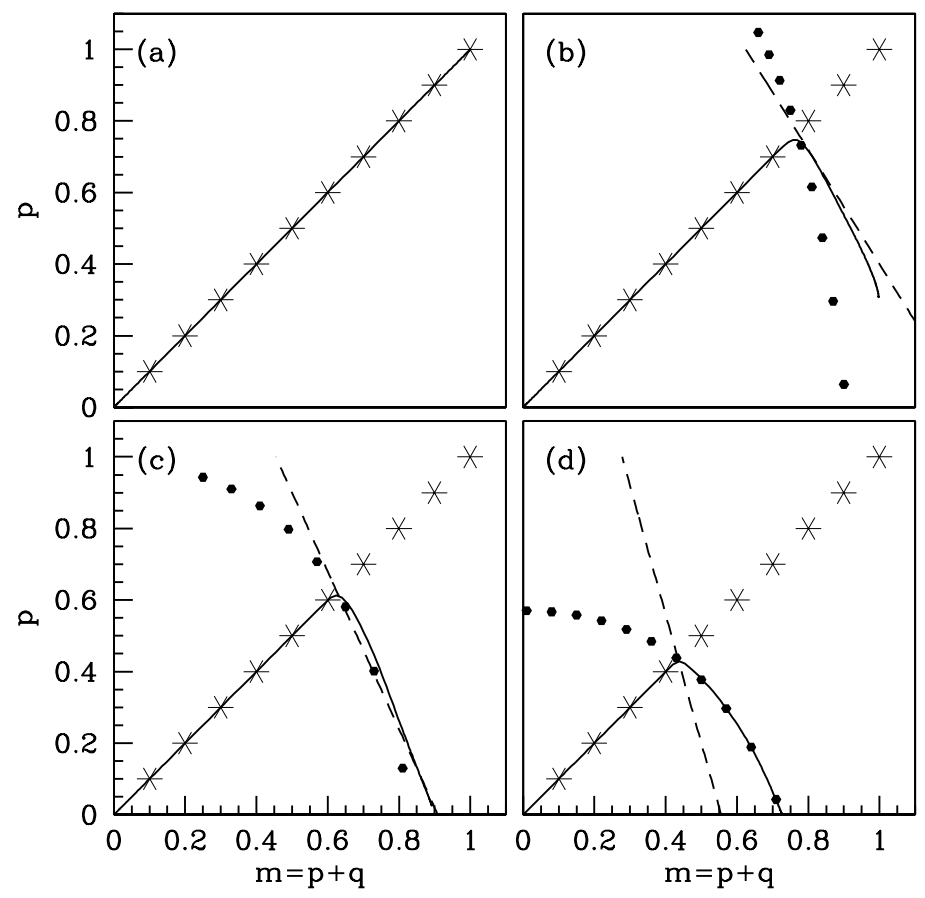

Fig. 7. Comparison between the analytical solution form derived in the main text for step function $f($.$) , and numerical solution of (3) for smooth f($.$) . The solid line ( -$ ) denotes the numerical solution, calculated with numerical details and end conditions as described in the legend to Figure 3. The dotted line $(\bullet \bullet \bullet)$ denotes the curve $P=\mathcal{H}(M)$, and the dashed line (- - - - -) denotes the line $\mathcal{L}$. The crosses $(*)$ denote the line $P=M$. The parameter values are $\gamma=2, c_{0}=1$ and $c^{*}=0.5$, which imply $\alpha_{\text {crit }} \approx 0.615$, with $\alpha$ having the value (a) 0.15 , (b) 0.9 , (c) 0.55 , (d) 0.4 . The function $f(c)=\frac{1}{2}[1-\tanh (100 c-50)]$, with $g(c) \equiv 1$ and $h(c)=\frac{1}{2} f(c)$. Note that since $f(c)$ is non-zero for all finite $c$ in this case, the solution will eventually develop a fully necrotic core (see §3.2). However, because $f($.) is very close to a step function, this is a long way behind the tumour edge, and has not begun to develop in the solution illustrated here. 
The condition for the tumour to develop a rim of proliferating cells is that case C or D applies, i.e.

$$
\alpha>1-c^{*} / c_{0} \text {. }
$$

The condition for the tumour to develop a proliferating rim, a quiescent band and a necrotic core is that case $\mathrm{D}$ applies, with $\alpha>1-c^{* *}$, that is

$$
\alpha>\max \left\{\alpha_{\text {crit }}, 1-c^{* *} / c_{0}\right\} .
$$

This is the structure familiar from work on multicellular spheroids in vitro.

When a proliferating rim develops, the maximum density of proliferating cells is given by the value of $P$ at which the line $P=M$ intersects the line $C=c^{*}$, namely

$$
P_{\text {max }}=\frac{\gamma\left(c_{0}-c^{*}\right)}{c_{0} \alpha \gamma+c^{*}} .
$$

This is a decreasing function of $c^{*}$, implying that the maximum density of proliferating cells goes down as the nutrient level required to switch on quiescence increases, as expected intuitively. Also, the maximum density increases with $\gamma=k_{1} / k_{2}$, since higher values of this ratio imply less nutrient uptake at given cell densities.

When a quiescent and/or necrotic core develops, the density of cells in this core is given by $M(-\infty)$. If case $\mathrm{D}$ applies, then $M(-\infty)$ is the solution of $\mathcal{H}(M)=0$, so that

$$
M(-\infty)+\log [1-M(-\infty)]=\log \left(1-\frac{\gamma\left(c_{0}-c^{*}\right)}{c_{0} \alpha \gamma+c^{*}}\right) .
$$

However, if case $\mathrm{C}$ applies, then $M(-\infty)=\left(1-c^{*} / c_{0}\right) / \alpha$. Thus $M(-\infty)$ decreases with $c^{*}$, and increases with $\gamma$ from the constant level $\left(1-c^{*} / c_{0}\right) / \alpha$, which applies for $\gamma<\gamma_{\text {crit }}$.

The width of the proliferating rim cannot be determined directly from the solution in the $P-M$ plane: this solution must be substituted back into (6a), giving a first order ODE for $P(z)$. The required integration cannot be done exactly for the part of the solution in which $\lambda=1$, which is the region of the solution behind the peak in proliferating cells. However, numerical solutions show that the pulse wave of $P$ is approximately symmetric about its peak, and thus an approximation to the width of this pulse can be found by considering the region in which $\lambda=0$. In this region, $P \equiv M$ and $C>c^{*}$, so that (6a) implies

$$
d P / d \zeta=P(1-P) \quad \Rightarrow P=1 /\left(1+k e^{-\zeta}\right)=1 /\left(1+k e^{+z / a}\right)
$$

where $k>0$ is an arbitrary constant of integration, corresponding to a translation in the wave. We take, as a simple measure of the width of the pulse wave, the distance between the point at which $P$ has its maximum value $P_{\text {max }}$ (defined in (12)) and the point with $P=\frac{1}{2} P_{\text {max }}$; this is easily calculated using (14) as

$$
\Delta z=a \log \left(1+\frac{1}{1-P_{\max }}\right)=a \log \left(\frac{(2+\gamma) c^{*}-(1-2 \alpha) \gamma c_{0}}{(1+\gamma) c^{*}-(1-\alpha) \gamma c_{0}}\right) \text {. }
$$

Note in particular that the proliferating rim becomes wider as the cell density within it increases. 


\section{Discussion}

The work presented in this paper makes three main contributions to the mathematical modelling of avascular tumour growth. Firstly, we have demonstrated the development of the characteristic layered structure of proliferating rim, quiescent band and necrotic core, within a PDE model encorporating cell movement. To the best of our knowledge, this is the first prediction of this structure within a model formulated in terms of continuous cell densities rather than discrete bands of the cell types, separated by moving boundaries calculated via oxygen concentration thresholds imposed a priori (as in Greenspan, 1972). Experimental evidence indicates gradual rather than sharp transitions between the layers (Hystad \& Rofstad, 1994), suggesting that our formulation may be more realistic.

Our second main contribution is the use of a new movement term incorporating contact inhibition of migration. Within the context of the model $(1,2)$, this term is not very important: the analysis in $\$ 3.2$ and $\$ 3.3$ shows that the development of the layered structure in our model solutions is essentially a function of the cell kinetics, depending on rates of proliferation, quiescence and necrosis as a function of nutrient level. However, the term becomes very significant when one extends the model to include the untransformed cells from surrounding tissue, whose density we denote by $s(x, t)$. Here we have in mind a tumour growing within an epithelium, with its growth inhibited by the presence of surrounding epithelial cells. These cells will themselves be motile, and will divide at a rate that depends on nutrient level, but that is intrinsically lower than that of proliferating tumour cells, by a factor $\Gamma<1$ say. Incorporating this new cell population gives the enlarged model

$$
\begin{aligned}
\frac{\partial p}{\partial t} & =\frac{\partial}{\partial x}\left[\frac{p}{p+q+s} \frac{\partial}{\partial x}(p+q+s)\right]+g(c) p(1-p-q-n-s)-f(c) p \\
\frac{\partial q}{\partial t} & =\frac{\partial}{\partial x}\left[\frac{q}{p+q+s} \frac{\partial}{\partial x}(p+q+s)\right]+f(c) p-h(c) q \\
\frac{\partial n}{\partial t} & =h(c) q \\
\frac{\partial s}{\partial t} & =\frac{\partial}{\partial x}\left[\frac{s}{p+q+s} \frac{\partial}{\partial x}(p+q+s)\right]+g(c) s(\Gamma-p-q-n-s) \\
\frac{\partial c}{\partial t} & =D_{c} \frac{\partial^{2} c}{\partial x^{2}}+k_{1} c_{0}[1-\alpha(p+q+n+s)]-k_{1} c-k_{2} p c-k_{3} s c .
\end{aligned}
$$

Numerical simulations of this enlarged model show qualitatively similar behaviour to that found in $(1,2)$ (Figure 8), with the surrounding tissue cells receding as the competing tumour cell population grows. However, the speed of tumour growth is significantly reduced by the inclusion of the surrounding cell population, as one expects intuitively, and this depends fundamentally on the use of the new movement term reflecting contact inhibition of migration. Calculation of the rate of tumour growth in this enlarged model is rather complex, and is discussed by Sherratt (2000) for a caricature model of the competition between the tumour and surrounding tissue. 


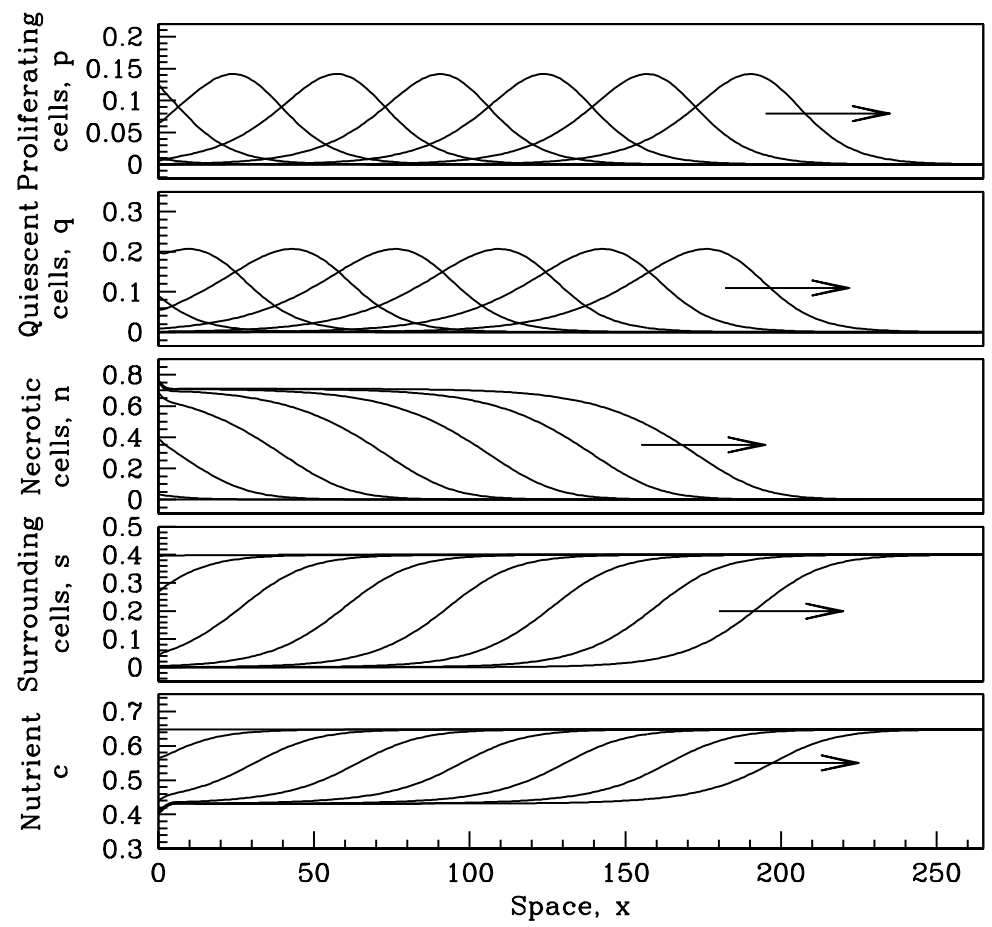

Fig. 8. Numerical solutions of the enlarged model equations (16), illustrating an avascular tumour growing within an epithelium. The solution has the form of an advancing pulse of proliferating cells $(p)$, with a band of quiescent cells $(q)$ and a necrotic core $(n)$ behind this; in parallel with this is a receding wave of surrounding tissue cells $(s)$ and also of nutrient (c). The solutions are plotted as a function of space at times $t=0,7.5,15, \ldots, 52.5$, with arrows indicating increasing time. Note that the solution has the same basic structure as that illustrated in Figure 2a, but that the speed of tumour growth is significantly reduced by the presence of surrounding tissue. The parameter values are as in Figure 2a, with $k_{3}=1$ and $\Gamma=0.4$. The initial conditions were $q \equiv n \equiv 0, c \equiv 1, p=0.01 \exp (-0.1 x)$, $s=\Gamma \cdot[1-0.01 \exp (-0.1 x)]$, and the boundary conditions used at $x=0$ and $x=265$ were $p_{x}=q_{x}=c_{x}=s_{x}=0$ (no boundary conditions are required for $n$ ). At $x=0$, this represents symmetry, but as in Figures 2 and 3, the boundary condition at $x=265$ is an artificial one, required because we cannot solve numerically on a semi-infinite domain.

The third main contribution of this work is the inclusion in the model of a term for nutrient supply from underlying tissue. Such an effect is clearly absent for multicellular spheroids grown in vitro, but is expected in the early stages of avascular tumour growth in vivo. Our results show that tumour structure can be significantly altered by this effect, to an extent that depends on parameters in a way that we have been able to quantify. Our results argue strongly for the development of a two-dimensional analogue of the multicellular spheroid assay as a key step in bridging the gap between in vitro experiments and tumour growth in vivo.

The results of our modelling work could be tested comprehensively using an approximately two-dimensional equivalent of the multicellular spheroid system, in 
which a sheet of tumour cells are grown on an appropriate substrate. Many model parameters are functions of the tumour cell line and cannot easily be altered, but crucially nutrient supply could easily be regulated, simply by altering the composition of the surrouding medium; in the model, this corresponds to varying $c_{0}$. For an experimental system of this kind, (10)-(15) are semi-quantitative predictions; they are not exact because of the assumption made in (7) on the form of $f(c)$. In particular, the model predicts that at high $c_{0}$, proliferating cells would be present throughout the tumour, with a proliferating rim developing at the critical value of $c_{0}$ implied by (10). A necrotic core would then develop as $c_{0}$ is reduced further until (11) is satisfied. The experimental system would also be able to test the predicted relationship, given in (15), between the width of the proliferating rim and the cell density within it. These experimental tests would provide an effective test of both the qualitative assumptions and quantitative details in the model.

\section{Appendix}

In this short Appendix, we show that equation (9) has a unique positive solution for $\alpha_{c r i t}$. This can be seen most easily by dividing through by $\alpha_{c r i t}^{2}$ and writing $y \equiv\left(1-c^{*}\right) / \alpha_{c r i t}$, giving the equation

$$
\begin{aligned}
\frac{\gamma\left(1-c^{*}\right)}{c^{*}}\left[1-\left(1-\frac{c^{*}}{\gamma\left(1-c^{*}\right)}\right) y\right] & =\Omega(y) \\
\text { where } \Omega(y) & =y^{2}\left(1-e^{-y}\right)^{-1} .
\end{aligned}
$$

The left hand side of (A.1) is a linear function of $y$, but can be increasing or decreasing depending on parameter values. Explicit differentiation shows that $\Omega^{\prime}(y)>0$ for $y>0$, with

$$
\begin{aligned}
\Omega^{\prime \prime}(y)= & \left(1-e^{-y}\right)^{-1}\left[2-4 \omega y+\omega y^{2}+2 \omega^{2} y^{2}\right] \\
& \text { where } \omega \equiv\left(1-e^{-y}\right)^{-1} e^{-y} \\
= & \left(1-e^{-y}\right)^{-1}\left[\left(2 \omega^{2}+\omega\right)\left\{y-\frac{2}{2 \omega+1}\right\}^{2}+\frac{2}{2 \omega+1}\right] \\
> & 0 \text { for all } y>0 .
\end{aligned}
$$

Therefore, $\Omega(y)$ is strictly increasing with strictly positive second derivative; moreover, $\Omega(0)=0$ while the left hand side of (A.1) is strictly positive at $y=0$. Therefore (A.1) has unique positive solution.

Acknowledgements. This work was supported in part by SHEFC Research Development Grants 107 (Centre for Theoretical Modelling in Medicine) and 099 (SIMBIOS Centre). JAS thanks Stephen Turner for helpful discussions. Permission from the copyright owner (American Association for Cancer Research, Inc.) and Prof. Wolfgang Mueller-Klieser (on behalf of the authors of Sutherland et al., 1986) to reprint their illustration of the structure of a multicellular spheroid (Fig. 1 in the present article) is gratefully acknowledged. 


\section{References}

Abercrombie, M.: Contact inhibition in tissue culture, In vitro., 6, 128-140 (1970)

Adam, J. A.: A simplified mathematical model of tumour growth, Math. Biosci., 81, 224-229 (1986)

Basjer, Z., Vuk-Pavlovic̀, S., Huzak, M.: Mathematical modeling of tumour growth kinetics. In: Adam, J. A., Bellomo, N. (eds.) A survey of models for tumor-immune system dynamics, Birkhäuser, Boston (1997)

Byrne, H. M., Chaplain, M. A. J.: Free boundary value problems associated with the growth and development of multicellular spheroids, Eur. J. Appl. Math., 8, 639-658 (1997)

Byrne, H. M.: A weakly nonlinear analysis of a model of avascular solid tumour growth, J. Math. Biol., 39, 59-89 (1999)

Canosa, J.: On a nonlinear diffusion equation describing population growth, IBM J. Res. Dev., 17, 307-313 (1973)

Casciari, J. J., Sotirchos, S. V., Sutherland, R. M.: Variations in tumor cell growth rates and metabolism with oxygen concentration, glucose concentration, and extracellular $\mathrm{pH}, \mathrm{J}$. Cell. Physiol., 15, 386-394 (1992)

Chaplain, M. A. J., Anderson, A. R. A.: Mathematical modelling, simulation and prediction of tumour-induced angiogenesis, Invasion \& Metastasis., 16, 222-234 (1996)

Chaplain, M. A. J., Britton, N. F.: On the concentration profile of a growth inhibitory factor in multicell spheroids, Math. Biosci.., 115, 233-243 (1993)

Chaplain, M. A. J., Stuart, A. M.: A model mechanism for the chemotactic response of endothelial cells to tumor angiogenesis factor, IMA J. Math. Appl. Med. Biol., 10, 149-168 (1993)

Davies, C. D., Muller, H., Hagen, I., Garseth, M., Hjelstuen, M. H.: Comparison of extracellular matrix in human osteosarcomas and melanomas growing as xenografts, multicellular spheroids, and monolayer cultures, Anticancer Res., 17, 4317-4326 (1997)

Durand, R. E.: Multicell spheroids as a model for cell kinetic studies, Cell Tissue Kinetics., 23, 141-159 (1990)

Fisher, R. A.: The wave of advance of advantageous genes, Ann. Eugenics., 7, 353-369 (1937)

Freyer, J. P., Sutherland, R. M.: Regulation of growth saturation and development of necrosis in EMT6/Ro multicellular spheroids by the glucose and oxygen supply, Cancer Res., 46, 3504-3512 (1986)

Freyer, J. P.: Decreased mitochondrial function in quiescent cells isolated from multicellular tumor spheroids, J. Cell Physiol., 176, 138-149 (1998)

Friedman, A., Reitich, F.: Analysis of a mathematical model for the growth of tumors, $J$. Math. Biol., 38, 262-284 (1999)

Grander, D.: How do mutated oncogenes and tumor suppressor genes cause cancer?, Medical Oncol., 15, 20-26 (1998)

Greenspan, H. P.: Models for the growth of a solid tumour by diffusion, Stud. Appl. Math., 51, 317-340 (1972)

Groebe, K., Mueller-Klieser, W.: On the relation between size of necrosis and diameter of tumor spheroids, Int. J. Rad. Oncol., 34, 395-401 (1996)

Hamilton, G.: Multicellular spheroids as an in vitro tumour model, Cancer Lett., 131, 29-34 (1998)

Henry, T. D.: Science, medicine, and the future - Therapeutic angiogenesis, Brit. Med. J., 318, 1536-1539 (1999)

Hystad, M. E., Rofstad, E. K.: Oxygen consumption rate and mitochondrial density in human melanoma monolayer cultures and multicellular spheroids, Int. J. Cancer., 57, 532-537 (1994) 
Keller, E. F., Segel, L. A.: Model for chemotaxis, J. Theor. Biol., 30, 225-234 (1971)

Kolmogoroff, A., Petrovsky, I.: \& Piscounov, N. Etude de l'équation de la diffusion avec croissance de la quantité de matière et son application à un problème biologique, Moscow Univ. Bull. Math., 1, 1-25 (1937)

Kunz-Schughart, L. A., Kreutz, M., Knuechel, R.: Multicellular spheroids: a three-dimensional in vitro culture system to study tumour biology, Int. J. Exp. Path., 79, 1-23 (1998)

McElwain, D. L. S. \& Pettet, G. J.: Cell migration in multicell spheroids: swimming against the tide, Bull. Math. Biol., 55, 655-674 (1993)

Mueller-Klieser, W.: Method for determination of oxygen consumption rates and diffusion coefficients in multicellular spheroids, Biophys. J., 46, 343-348 (1984)

Mueller-Klieser, W., Freyer, J. P., Sutherland, R. M.: Influence of glucose and oxygen supply conditions on the oxygenation of multicellular spheroids, Br. J. Cancer., 53, 345-353 (1986) Nederman, T., Norling, B., Glimelius, B., Carlsson, J., Brunk, U.: Demonstration of an extracellular matrix in multicellular tumour spheroids, Cancer Res., 44, 3090-3097 (1984) Paulus, W., Huettner, C., Tonn, J. C.: Collagens, integrins and the mesenchymal drift in glioblastomas: a comparison of biopsy specimens, spheroid and early monolayer cultures, Int. J. Cancer., 58, 841-846 (1994)

Please, C. P., Pettet, G. J., McElwain, D. L. S.: Avascular tumour dynamics and necrosis, Math. Models Methods Appl. Sci., 9, 569-580 (1999)

Schwachofer, J. H. M., Acker, H., Crooijmans, R. P. M. A., Vangasteren, J. J. M., Holtermann, G., Hoogenhout, J., Jerusalem, C. R., Kal, H. B.: Oxygen tensions in two human tumor cell lines grown and irradiated as multicellular spheroids, Anticancer Res., 11, 273-279 (1991) Sherratt, J. A.: Wave front propagation in a competition equation with a new motility term modelling contact inhibition between cell populations, Proc. R. Soc. Lond., A 456, 2365$2386(2000)$

Sherratt, J. A., Murray, J. D.: Models of epidermal wound healing, Proc. R. Soc. Lond., B 241, 29-36 (1990)

Shymko, R. M., Glass, L.: Cellular and geometric control of tissue growth and mitotic instability, J. Theor. Biol., 63, 355-374 (1976)

Sutherland, R. M., McCredie, J. A., Inch, W. R.: Growth of multicell spheroids in tissue culture as a model of nodular carcinomas, J. Natl. Cancer Inst., 46, 113-120 (1971)

Sutherland, R. M., Sordat, B., Bamat, J., Gabbert, H., Bourrat, B., Mueller-Klieser, W.: Oxygenation and differentiation in multicellular spheroids of human colon carcinoma, Cancer Res., 46, 5320-5329 (1986)

Sutherland, R. M.: Cell and environment interactions in tumour microregions: the multicell spheroid model, Science, 240, 177-184 (1988)

Thomlinson, R. H., Gray, L. H.: Histological structure of some human lung cancers and the possible implications for radiotherapy, Brit. J. Cancer., 9, 539-549 (1955)

Ward, J. P., King, J. R.: Mathematical modelling of avascular tumour growth, IMA J. Math. Appl. Med. Biol., 14, 39-70 (1997)

Ward, J. P., King, J. R.: Mathematical modelling of avascular tumour growth, IMA J. Math. Appl. Med. Biol., 16, 171-211 (1999)

Weinberg, R. A.: Tumour suppressor genes, Science, 254, 1138-1146 (1991) 